\title{
Unfolding and Validation of SREM Fluxes
}

\author{
I. Sandberg, I. A. Daglis, A. Anastasiadis, P. Bühler, P. Nieminen, and H. Evans
}

\begin{abstract}
The Standard Radiation Environment Monitor (SREM) belongs to a second generation of instruments in a program established by the European Research and Technology Centre of the European Space Agency (ESA) to provide minimum intrusive particle radiation detectors on ESA spacecraft for space weather applications, which are also suitable for scientific investigations.

SREM detects high-energy electrons and protons and bins the measurements in fifteen counters characterized by overlapping energy bands, resulting in the appearance of strong correlation and contamination effects among the binned count-rates.

In this work a novel method to unfold measurements of SREM, i.e., to convert SREM counts to fluxes, is presented. The method is based on the derivation of multiple solutions of the inverse problem over various proton and electron energy ranges using the powerful technique of the Singular Value Decomposition (SVD).

Results and validation studies for measurements of Solar Energetic Particle Events are also presented.
\end{abstract}

Index Terms-Deconvolution, inverse problems, protons, radiation monitoring, semiconductor radiation detectors, solar system, space charge, sun.

\section{INTRODUCTION}

$\mathbf{T}$ HE ESA Standard Radiation Environment Monitor (SREM) [1] belongs to a second generation of instruments in a program established by the European Research and Technology Centre of the European Space Agency (ESA) to provide minimum intrusive particle radiation detectors on ESA spacecraft for space weather applications. SREM units monitor the radiation environment and provide suitable functions related to hazard for the host spacecraft and its payload. An alarm flag can be set whenever high radiation levels are reached during the spacecraft's orbit and the payload instruments may react accordingly entering, if necessary, in a special safe mode that protects them from possible radiation damages.

SREM was designed to measure electrons with energies $E>$ $0.5 \mathrm{MeV}$ and protons with energies $E>10 \mathrm{MeV}$ with fair spectral and angular resolution. Seven SREM units have been

Manuscript received September 15, 2011; revised January 13, 2012; accepted February 01, 2012. Date of publication March 02, 2012; date of current version August 14, 2012

I. Sandberg, I. A. Daglis, and A. Anastasiadis are with the Institute for Space Applications and Remote Sensing, National Observatory of Athens, 15236 Penteli, Greece (e-mail: sandberg@noa.gr; daglis@noa.gr; anastasi@noa.gr).

P. Bühler is with the Paul Scherrer Institute, 1130 Vienna, Austria (e-mail: paul.buehler@psi.ch).

P. Nieminen and H. Evans are with the ESTEC, European Space Agency, 2200 AG Noordwijk, The Netherlands (e-mail: petteri.nieminen@esa.int; hugh. evans@esa.int).

Color versions of one or more of the figures in this paper are available online at http://ieeexplore.ieee.org.

Digital Object Identifier 10.1109/TNS.2012.2187216 already launched on-board STRV-1C, Proba-1, INTEGRAL, Rosetta, GIOVE-B, Herschel and Planck spacecraft.

SREM consists of three silicon detectors. As charged particles traverse the Si crystal, they interact with the valence band electrons and promote them to the conduction band, where the carriers are free to move in response to an externally applied electric field. The resulting current, which is proportional to the energy loss of the incident particles is fed into a charge-sensitive pre-amplifier that converts it into a voltage pulse. The pre-amplified pulses are scrutinized by fast comparators, and counted into 15 detector channels. Each channel has its own characteristic proton and electron energy response. As a consequence of this scheme, proton and electron events counted in each channel are mixed. In addition, events recorded in high-energy channels appear also in channels with lower energy thresholds.

SREM provides reliable measurements for characterization of space radiation environment [2]-[4]. However, in all previous studies based on SREM data the environment was described either in terms of SREM count-rates or in terms of particle fluxes in rather wide energy windows. This is attributed to the reason that the SREM counts to flux calculation is not trivial as the associated inversion is unstable. There are several conversion methods that have been considered which provide a first estimate on the fluxes. A standard approach is to assume a parametric form for the particle fluxes and apply standard minimization techniques, such as the maximum likelihood or the least squares fit, to estimate the values of the free parameters. However, this method is limited since it requires an assumption of the spectral form and as a consequence it can provide successful results only when the assumed spectral form fits to the measured spectra. The simple conversion factor (SCF) method is based on the mean of the integral transform of the response function with a sampling of space environment spectra [3]. This method is applicable only for count-rates attributed solely to a single particle species, i.e., protons or electrons, and allows a fast estimation of the fluxes within the wide sensitivity energy ranges of SREM counters.

For the efficient conversion of SREM counts to charged particle fluxes with high spectral resolution we have developed a novel method. The method is based on the Singular Value Decomposition (SVD) technique but includes additional schemes that permits the derivation of smooth and hard spectrum over large energy ranges. In order to validate the results of the developed method, we have unfolded several SREM measurements from the unit on board INTEGRAL associated with measurements of Solar Energetic Particle Events (SEPEs) and compared them with the Solar Energetic Particle Environment Modelling (SEPEM) dataset [5] which includes well-processed, inter-calibrated, cleaned dataset of SEPEs based on measurements from different scientific instruments. The comparisons indicate that 
TABLE I

LIST OF THE SREM CHANNELS AND THE CORRESPONDING ENERGY RANGES of Detected Protons and Electrons [3]

\begin{tabular}{|l||cc||cc|}
\hline SREM & Proton Energy & {$[\mathrm{MeV}]$} & Electron Energy & {$[\mathrm{MeV}]$} \\
\hline Bin & $E_{\min }$ & $E_{\max }$ & $E_{\min }$ & $E_{\max }$ \\
\hline \hline TC1 & 27 & $\infty$ & 2.00 & $\infty$ \\
S12 & 26 & $\infty$ & 2.08 & $\infty$ \\
S13 & 27 & $\infty$ & 2.23 & $\infty$ \\
S14 & 24 & 542 & 3.20 & $\infty$ \\
S15 & 23 & 434 & 8.18 & $\infty$ \\
\hline TC2 & 49 & $\infty$ & 2.80 & $\infty$ \\
S25 & 48 & 270 & - & - \\
C1 & 43 & 86 & - & - \\
C2 & 52 & 278 & - & - \\
C3 & 76 & 450 & - & - \\
C4 & 164 & $\infty$ & 8.10 & $\infty$ \\
\hline TC3 & 12 & $\infty$ & 0.80 & $\infty$ \\
S32 & 12 & $\infty$ & 0.75 & $\infty$ \\
S33 & 12 & $\infty$ & 1.05 & $\infty$ \\
S34 & 12 & $\infty$ & 2.08 & $\infty$ \\
\hline
\end{tabular}

the developed method is rather successful and allows the reconstruction of reliable proton spectra with the highest spectral resolution compared to all available methods used for SREM data so far.

\section{Characteristics OF THE SREM InStRUMENT}

The SREM unit consists of three silicon diode detectors (D1, D2 and D3) in a two-detectors-head configuration. One system is a single silicon diode detector (D3) and the other system uses two silicon diodes (detectors D1/D2) one after the other, in a co-axial configuration (detector telescope configuration). Each SREM unit is contained in a box of $20 \times 12 \times 10 \mathrm{~cm}^{3}$ and weighs $2.6 \mathrm{~kg}$.

The aperture of the detector has an opening angle of \pm 20 degrees. However, ultra high energy particles may penetrate from any direction. For the coincidence counters the acceptance is essentially restricted by the aperture. The detector electronics are capable of processing a detection rate of 100 $\mathrm{kHz}$ with dead-time correction below $20 \%$. The main entrance of the D3 window is covered with $0.7 \mathrm{~mm}$ aluminum layer, which defines the lower energy threshold for electrons to $E>0.5 \mathrm{MeV}$ and for protons to $E>10 \mathrm{MeV}$. The D1/D2 detector is covered with a $2 \mathrm{~mm}$ aluminum layer giving a proton and electron threshold of 20 and $1.5 \mathrm{MeV}$, respectively. The diodes of the telescope configuration are separated by a $1.7-\mathrm{mm}$-thick aluminum and $0.7 \mathrm{~mm}$ thick tantalum layer. In addition, the shielding between the two diodes in the telescope prevents the passage of electrons. However, protons with energies greater than $43 \mathrm{MeV}$ go through, depositing energy in both detectors. Thus, using the two diodes in coincidence gives pure proton count-rates. All pre-amplified detector pulses are scrutinized by a set of fifteen fast comparators (channels) - eleven for single events and four for coincidences - providing energy spectral information (Table I). SREM channels identify particle energies by the energy deposited in the detector.

SREM has been calibrated with protons at the Proton Irradiation Facility (PIF) of the Paul Scherrer Institute and electrons from radioactive sources such as ${ }^{90} \mathrm{Sr}$ and ${ }^{60} \mathrm{Co}$ [6]. In addition. the measurements were simulated with the Monte Carlo code GEANT3 from CERN [7] and a good agreement between

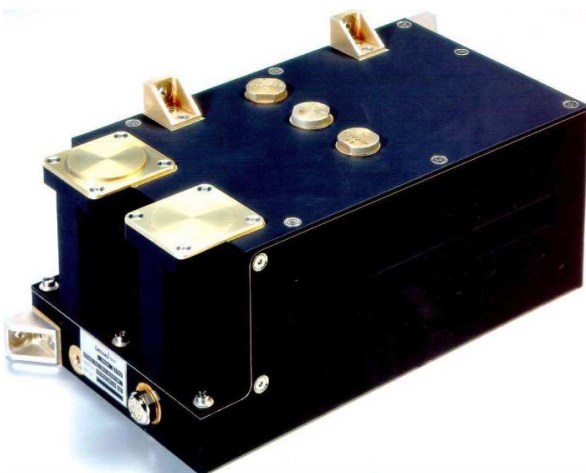

Fig. 1. The SREM unit on-board the INTEGRAL mission.
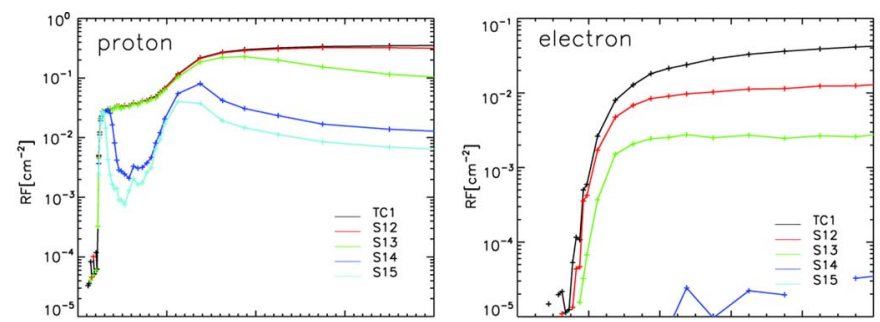

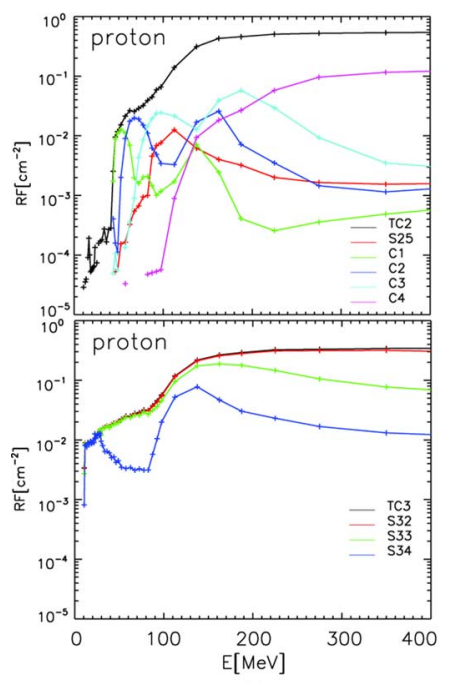

(a)

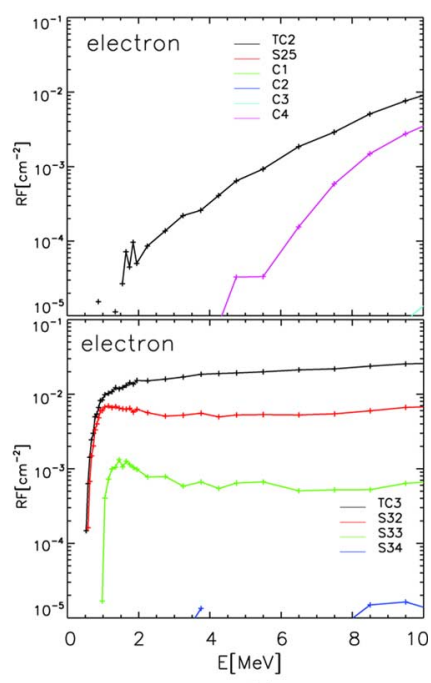

(b)
Fig. 2. The proton (left column) and the electron (right column) response functions $R F_{i, q}(E)$ of the INTEGRAL/SREM unit for the D1, D1/D2 and D3 group of counters.

measurements and simulations was found. The response matrix RF for each SREM unit has been derived by simulations of omni-directional fluxes of electrons and protons using the GEANT SREM model which was supplemented with a mass model of the corresponding host spacecraft. In Fig. 2, we present the response functions $R F_{i, q}(E)$ for the INTEGRAL/SREM unit [8].

The minimum energy in the "total count-rate" channels of detectors 1 and 3 (TC1 and TC3) correspond to the threshold penetration of the shields. All the channels are sensitive to protons, while the coincidence channels C1-C4, S25 and the S15 can be considered as pure proton channels (see Table I). However, in the presence of high-energy electrons (with energies above $E>8 \mathrm{MeV}$ ) also $\mathrm{S} 15$ and $\mathrm{C} 4$ can be contaminated by electron counts. Obviously, there are no pure electron channels. Note, 
that unlike spectrometers, which are able to measure incident spectra in narrow distinct energy intervals, SREM samples the spectra in broad and overlapping energy bands; e.g., channels TC1, S12 and S12 (or TC3, S32, S33 and S34). As a consequence, the count-rates of the channels are strongly dependent on each other; e.g., counts in S15 are also counted in S14, S13, S12 and TC1.

\section{UNFOLDING SREM COUNTS}

The measured count-rates in SREM counter $C_{i}, i=$ $1 \ldots N_{b}(=15)$ are given by the sum

$$
C_{i}=\sum_{q=p, e} \int_{0}^{\infty} f_{q}(E) R F_{i, q}(E) d E
$$

where each term of the sum is attributed to the incident proton and electron fluxes. Here, $f_{q}(E)$ denotes the omni-directional fluxes in $\left[\mathrm{cm}^{-2} \mathrm{MeV}^{-1} \mathrm{~s}^{-1}\right]$ units and $R F_{i, q}(E)$ the corresponding response function for $q=p, e$. The calculation of $f_{q}(E)$ requires the inversion of (1). This equation is a Fredholm integral equation of the first kind and is a classical example of an ill-posed problem as its solution $f_{q}(E)$ is not unique and not a continuous function of the counts $C_{i}$.

If we consider a step function form for the charged particle fluxes, and discretize into $N_{p}\left(N_{e}\right)$ the proton (electron) flux and energy levels the integral equation takes the form of a linear system of equations which can be written in the following matrix form $\mathbf{C}=\mathbf{R F} \cdot \overline{\mathbf{f}}$. Here $\mathbf{C}$ is a vector containing the measured count-rates, $\overline{\mathbf{f}}=\mathbf{f} \boldsymbol{\Delta} \mathbf{E}$ is a vector containing the $N_{x}=$ $N_{p}+N_{e}$ values of the unknown differential fluxes $\mathbf{f}=\left[\mathbf{f}_{\mathbf{p}}, \mathbf{f}_{\mathbf{e}}\right]$ and $\mathbf{R F} \in \mathcal{R}^{N_{b} \times N_{x}}$ contains the elements of the response matrix for the selected $N_{x}$ energy bins.

The measured fluxes change by several orders of magnitude over the range of energies covered by the SREM device. Thus, a logarithmic binning is applied to the proton and electron energy ranges. The averaged values of the response matrix are considered, which are calculated via a numerical integration within the energy range of each bin. Errors attributed to the discretization of the energies and to the uncertainties of the calculated response functions are not taken into account.

In order to give equal weight to all equations of the linear system $\mathbf{C}=\mathbf{R F} \cdot \overline{\mathbf{f}}$, we rescale the individual equations according to their statistical significance by dividing the elements of $\mathbf{C}$ and $\mathbf{R F}$ with the square root of the associated count-rate. This transformation preserves the form of the system of equations leaving the unknown vector $\overline{\mathbf{f}}$ unchanged. For the sake of simplicity, the same notations to the untransformed system are kept.

The linear system can be numerically solved and the flux levels can be estimated. However, such a system of equations belongs to the class of discrete ill-posed problems which are unstable against small variations due to the limited resolution of the detector and the uncertainties of the measurements. As a consequence its numerical solution is widely oscillating (presents large variations of fluxes in neighboring energy bins) and may receive unphysical negative values for particle fluxes. There are various techniques that are being used, e.g., in the field of high energy particle physics [9], allowing unfolding of folded distributions from (1).

In what follows, we present an unfolding method which uses the efficient and fast semi-analytical approach presented in [10] as a starting point. However, the direct application of this approach for the unfolding of SREM data is not successful due to the strongly overlapping energy bands, the electron-proton contamination effects and the fact that the measured spectra decay over logarithmic scales.

\section{A. SVD Approach to Data Unfolding}

In order to avoid the oscillatory behavior of the numerical solution of equation $\mathbf{C}=\mathbf{R F} \cdot \overline{\mathbf{f}}$, we follow the work of [10], and we consider the following regularized system of equations

$$
\left[\begin{array}{c}
\mathbf{R F} \\
\sqrt{\tau} \mathbf{R}
\end{array}\right] \cdot \overline{\mathbf{f}}=\left[\begin{array}{l}
\mathbf{C} \\
\mathbf{0}
\end{array}\right]
$$

which introduces a regularisation condition through the inclusion of the matrix $\mathbf{R}$ that allows the solution to be smooth, with small bin-to-bin variation. The regularisation minimizes a priori the curvature-defined here as the difference in the derivatives between two successive energy bins, i.e., $\gamma_{i}=\bar{f}_{i-1}-2 \bar{f}_{i}+\bar{f}_{i+1}$. As a consequence the unwanted oscillations are suppressed.

Matrix $\mathbf{R}$ has non-zero elements only in the main and the first diagonals: $R_{i, i}=-2, R_{i+1, i}=1$, for $i \neq 1, N_{x}$. The first $N_{p}$ columns regularize the proton flux levels, while the last $N_{e}$ columns regularize the electron flux levels. For the edge points of the proton and the electron spectra the curvature cannot be defined and the suppression of large differences $\gamma_{i}=\bar{f}_{i}-$ $\bar{f}_{i-1}$ or $\gamma_{i}=\bar{f}_{i+1}-\bar{f}_{i}$ is required instead by setting $R_{i, i}=$ $-1, R_{i-1, i}=1, R_{i-1, i}=0$ for $i=1, N_{x}$ and $R_{i, i}=-1$, $R_{i-1, i}=1, R_{i-1, i}=0$ for $i=N_{p}, N_{p}+1$ since the proton and electron spectra are independent functions. The parameter $\tau$ determines the relative weight of the regularisation condition and is termed as the regularisation parameter.

The regularized system of equations can be solved using basic properties of the Singular Value Decomposition (SVD) technique. The decomposition of the product $\mathbf{R F} \cdot \mathbf{R}^{-\mathbf{1}}=\mathbf{U S V}^{\mathbf{T}}$ into its left $\mathbf{U} \in \mathcal{R}^{N_{b} \times N_{b}}$, right $\mathbf{V} \in \mathcal{R}^{N_{x} \times N_{x}}$ singular vectors, and the $\mathbf{S} \in \mathcal{R}^{N_{b} \times N_{x}}$ diagonal singular value matrix (with positive diagonal elements $s_{i}$ in descending order) permits the analytic expression of the solution of the regularized equations in the following form [10]:

$$
\overline{\mathbf{f}}=\mathbf{R}^{-\mathbf{1}} \mathbf{V} \mathbf{z}, \text { where } z_{i}=\frac{d_{i} s_{i}}{s_{i}^{2}+\tau} .
$$

It should be noted that the matrix $\mathbf{R}$ is degenerate and cannot be inverted. Thus, a small numerical value 0.001 has been added to the non-zero elements of $\mathbf{R}$ prior to its numerical inversion.

Note, that the covariance matrix of the solution can be consistently calculated and is given [10] by

$$
\operatorname{cov}(\overline{\mathbf{f}})=\mathbf{R}^{-\mathbf{1}} \mathbf{V Z V}^{\mathbf{T}} \mathbf{R}^{\mathbf{T}-\mathbf{1}}, \text { where } Z_{i k}=\frac{s_{i}^{2} \delta_{i k}}{\left(s_{i}^{2}+\tau\right)^{2}},
$$

providing the corresponding error estimates (error bars). 
The rank $k$ of the system (2) is actually equal to the number of significant singular values. The regularized solution may suppress the unwanted unphysical oscillatory depending on the value of the parameter $\tau$ with respect to $s_{k}$. For $\tau \ll s_{k}^{2}$ the impact of the regularisation is negligible, and as a consequence the solutions remain oscillatory and thus unacceptable. For $\tau \gg s_{k}^{2}$ the solutions get over-smoothed leading to a large bias. As it can be seen from (3) physically meaningful values of $\tau$ belong in the interval $s_{1}^{2}>\tau>s_{N_{b}}^{2}$. However, only a proper selection of $\tau$ may regularize the singularities - small $s_{i}$ 's-which appear for large values of index $i$ acting as a cut-off low pass Fourier filter.

There have been several suggestions for the optimum selection of the regularisation parameter. For histograms with large number of bins and smooth distributions, the effective rank of the system $k$ can be estimated graphically and used as an optimum choice for the regularisation parameter, $\tau=s_{k}^{2}$, providing smooth solutions with a sufficiently small bias [10]. We have tested the suggestions presented in the papers by [10] and [11] using SREM measurements associated to SEPEs. However, the derived spectra were unphysical, as they were receiving either negative values or they were strictly monotonically increasing. In the majority of all the studied cases, it turns out that the minimum regularisation parameter that can provide positive and smooth spectra through (3) receives values within intervals bounded by the largest squares of the singular values $s_{i}^{2}$. As a consequence, the regularized solution is expected to be significantly suppressed according to (3). The relatively large value of the minimum regularisation parameter reflects the large degeneration degree of the particular inverse problem attributed to the characteristics of the response of SREM units.

Any rescaling of the unknown equations that changes the distribution of the singular values of the system might lead to better estimates for the spectra. In reference [10] it is proposed that the response matrix should be rescaled with the expected values of the solutions - as calculated independently through Monte Carlo (MC) simulations studies. However, MC simulations for the unfolding of a continuous flow of SREM data attributed to events of different size and characteristics is not possible. Since we expect the flux levels to decrease in logarithmic scales with energy, we have selected to re-scale the regularisation matrix $\mathbf{R}$ by multiplying its elements with the width of the corresponding energy bins. In this case, the bin-to-bin variations in the regularized solutions are smoothed-out by suppressing a curvature defined here by $\gamma_{i}=f_{i-1}\left(\Delta E_{i-1}\right)^{2}-2 f_{i}\left(\Delta E_{i}\right)^{2}+$ $f_{i+1}\left(\Delta E_{i+1}\right)^{2}$.

For the selection of $\tau$, we perform a scanning in the range $s_{N_{b}}^{2}<\tau<s_{1}^{2}$ and choose as optimum value the smallest that provides positive and decaying values of the differential spectra for the first $N_{p}-i_{f}\left(N_{e}-i_{f}\right)$ out of $N_{p}\left(N_{e}\right)$ discrete proton (electron) energy levels. The right edge $i_{f}=1,2$ flux levels in the spectrum may present significant oscillations due to insufficient edge regularisation and/or due to the relatively poor statistics. Thus, we do not include them in the selection criteria of $\tau$ in order to decrease the suppression of the overall solution. The particular selections in the regularisation scheme turn to be rather useful as they permit us to derive smooth spectra that reconstruct sufficiently the measured count-rates. However, the selection of the total proton and electron energy ranges turn to be rather essential parameters for the hardness of the derived spectra.

\section{B. The Selection of Energy Ranges}

A natural choice for the selection of the total proton and electron energy range for the spectra to be unfolded would be the detection energy ranges of SREM units. However, the selection of a wide energy range - with respect to the characteristic energy of the true spectrum - may lead to significant suppression at the low energy flux levels of the derived spectrum as a result of the regularisation over (wide) energy ranges that might have relatively insufficient contribution to the total number of the measured count-rates per counter. This is particularly true for hard proton spectra and especially for the proton counters that present a local maximum in their response function before $E_{p}=200 \mathrm{MeV}$ (cf. Fig. 2). On the contrary, the selection of a relatively narrow energy range for the electron spectrum may lead to the derivation of an erroneous proton spectrum as a significant part of the measured count-rates originated from incident electrons within the excluded energy ranges may be attributed to the protons.

It is evident that the selection of the total proton and electron energy range turns to be critical since the degree of oversmoothing depends on the selected energy range for the solution with respect to the characteristic range of the measured spectrum. In order to overcome this inherent problem we derive multiple proton (electron) solutions by applying the SVD method described in the previous section using multiple upper limits in the proton (electron) energy range and keeping fixed the electron (proton) range each time. The measured proton (electron) spectrum is reconstructed by choosing among the solutions the maximum flux value for each proton (electron) energy bin.

In Fig. 3, we present as an example results of the analysis of the INTEGRAL/SREM measurements of January 20, 2005, 12:00 UT associated with a large SEPE. The set of the dotted lines represent different solutions for the proton spectra, according to (3), derived by varying the total number of proton energy bins - i.e by considering different values for the right bound of the proton energy range - and using the regularisation scheme presented in previous subsection.

As the width of the energy range increases, the proton spectrum gets suppressed due to the regularisation. Note that the difference in the lowest energy flux levels is of the order of 5 in the particular example. For the reconstruction of the measured proton spectrum we select for each energy level the maximum value found in the family of the solutions (red lines in Fig. 3). In limited cases, the sum of the reconstructed count rates $\sum_{i=1}^{15} C_{i}^{r e c}$ derived by folding the reconstructed proton spectrum with the proton response function is slightly larger compared to the sum of the original count-rates. In these cases, a small correction factor $\sum_{i=1}^{15} C_{i} / \sum_{i=1}^{15} C_{i}^{r e c}$ can be applied in the flux levels of the proton spectrum. This change shifts the spectrum slightly downwards without modifying its shape. This approach allows us to derive non-suppressed solutions within a wide energy range by using the inherently suppressed smooth regularised solutions. 


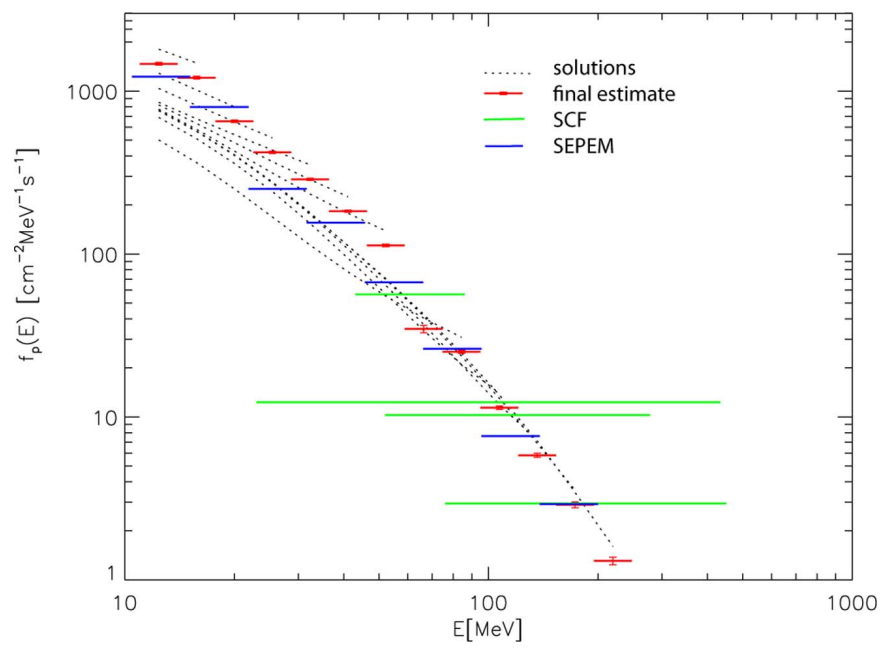

Fig. 3. Estimation of proton spectra (red lines) using the SVD solutions for different energy windows (black dotted lines). The flux levels derived with the SCF method (green lines) and from the SEPEM proton dataset (blue lines) are plotted for comparison.

The green and the blue lines in Fig. 3 denote the flux levels derived using the SCF method [3] and the associated SEPEM [5] dataset values interpolating at the INTEGRAL/SREM measurements times. It is evident that the level of agreement with the reconstructed spectrum is much better compared to any of the individual solutions.

\section{RESUlTS AND COMPARISONS}

In the following, we present results of the method described above using very large datasets of INTEGRAL/SREM measurements associated with two of the largest sequence of SEPEs that occurred during the last solar cycle. In order to validate our findings, we have performed extensive comparisons with SEPEM dataset [5].

In Fig. 4, we present the INTEGRAL/SREM count rates associated to the large SEPEs that took place during January 2005. The enhancements in the measurements just before January 19 and 22 are attributed to the crossings of spacecraft through the outer Radiation Belt. Using the steps described in the previous section we have calculated the charged particle fluxes based on the SREM measurements presented in Fig. 4 and using the INTEGRAL/SREM response function (cf. Fig. 2). The total proton energy was ranging up to $400 \mathrm{MeV}$ and was logarithmically split to $N_{p}=15$ bins, while the selected total electron range was ranging up to $15 \mathrm{MeV}$ and was logarithmically binned to $N_{e}=10$ values.

A necessary condition that should be hold for a successful evaluation of the derived fluxes is the agreement between the measured and the reconstructed count-rates. The latter ones can be calculated by folding the derived fluxes with the response matrix according to (1). In Fig. 5, we present a comparison between the count-rate datasets by using a scatter plot of $7277 \times 15$ out of $9473 \times 15$ points for the fifteen SREM counters.

The missing points include (manually extracted) measurements within the Radiation Belts and measurements characterized by low intensity. For the characterization of low in-

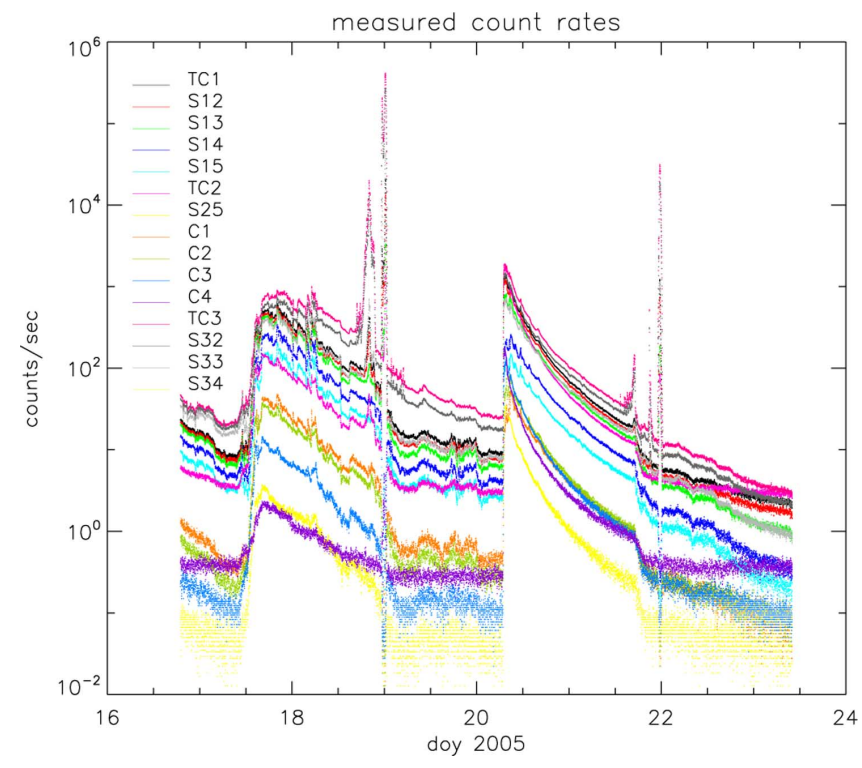

Fig. 4. Measurements of INTEGRAL/SREM for the January 2005 SEPEs, versus the Days of Year (DOY) 2005.

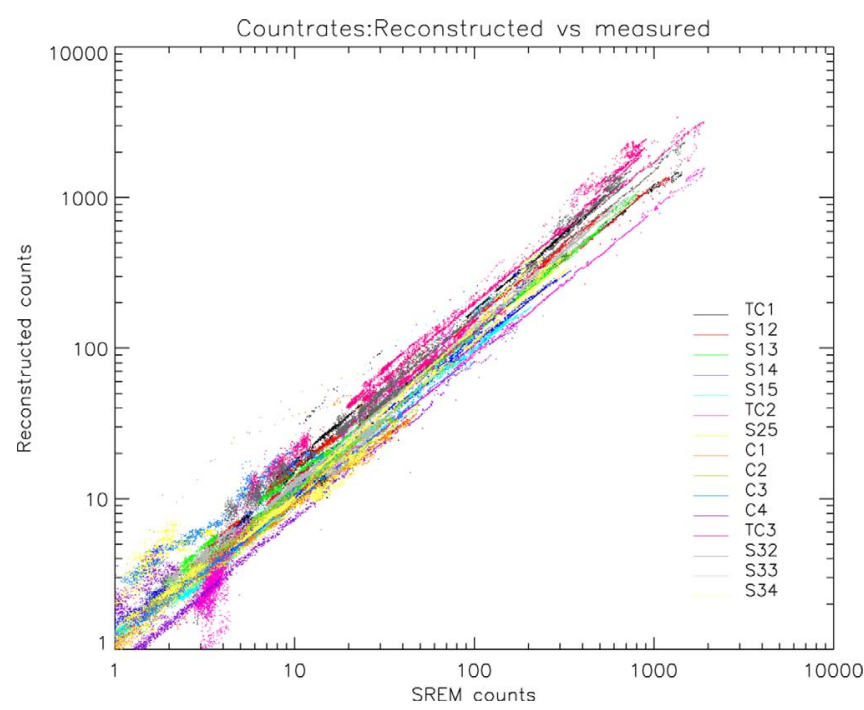

Fig. 5. Reconstructed count-rates derived by folding the SVD based derived fluxes for the January 2005 SEPEs with the INTEGRAL/SREM response matrix function versus the measured ones.

tensity we have set as a threshold the omni-differential proton flux not to exceed the $10 \mathrm{p}^{+} \mathrm{MeV}^{-1} \mathrm{~cm}^{-2} \mathrm{~s}^{-1}$ within the energy bin $[10.5,15.1] \mathrm{MeV}$ which is logarithmically centered at 12.6 $\mathrm{MeV}$. Note, that this filtering criterion has been applied to all the scatter plots presented throughout this paper.

In Fig. 6, we present the unfolded proton flux series interpolated at the logarithmic centers of the proton energy bins used in the SEPEM dataset. The proton flux series of the SEPEM dataset are presented in Fig. 7.

Before proceeding to the comparisons between the derived fluxes and the SEPEM dataset one it is necessary to verify that the SREM/INTEGRAL count-rate dataset matches well with the SEPEM flux dataset. This can be achieved independently from the counts to flux inversion method by comparing the INTEGRAL/SREM data with the SEPEM/SREM count-rates de- 


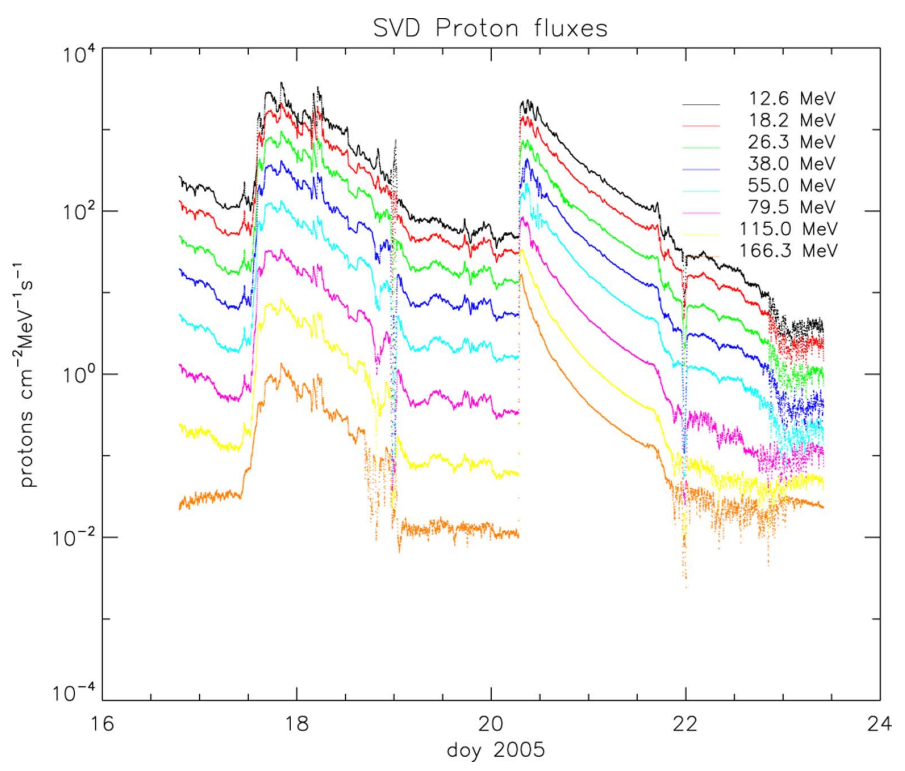

Fig. 6. The SVD-derived INTEGRAL/SREM proton flux series interpolated at selected SEPEM energy bins for the January 2005 SEPEs.

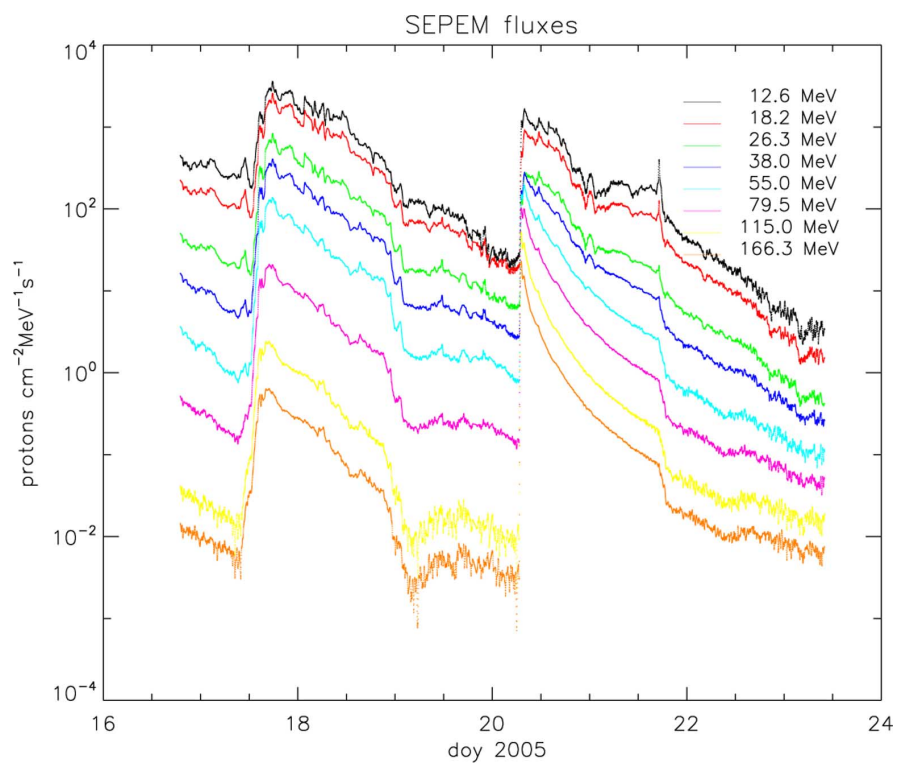

Fig. 7. Proton flux series of the SEPEM dataset for the January 2005 SEPEs for proton energies above $10 \mathrm{MeV}$.

rived by interpolating the SEPEM fluxes at INTEGRAL/SREM measurements times and folding the resulted SEPEM fluxes with the SREM response function. In Fig. 8 we present a scatter plot which shows a consistency between the count-rate datasets. Since the SEPEM/SREM counts are attributed solely to proton fluxes it is easy to conclude that the electron contribution (outside the filtered Radiation Belts) in the SREM data for the particular event is not dominant.

Provided the association of the measured count-rates with both reconstructed INTEGRAL/SREM (cf. Fig. 5) and SEPEM/ SREM count-rates (cf. Fig. 8) it is meaningful to perform the comparison between the unfolded and the SEPEM proton fluxes using the scatter plot presented in Fig. 9.

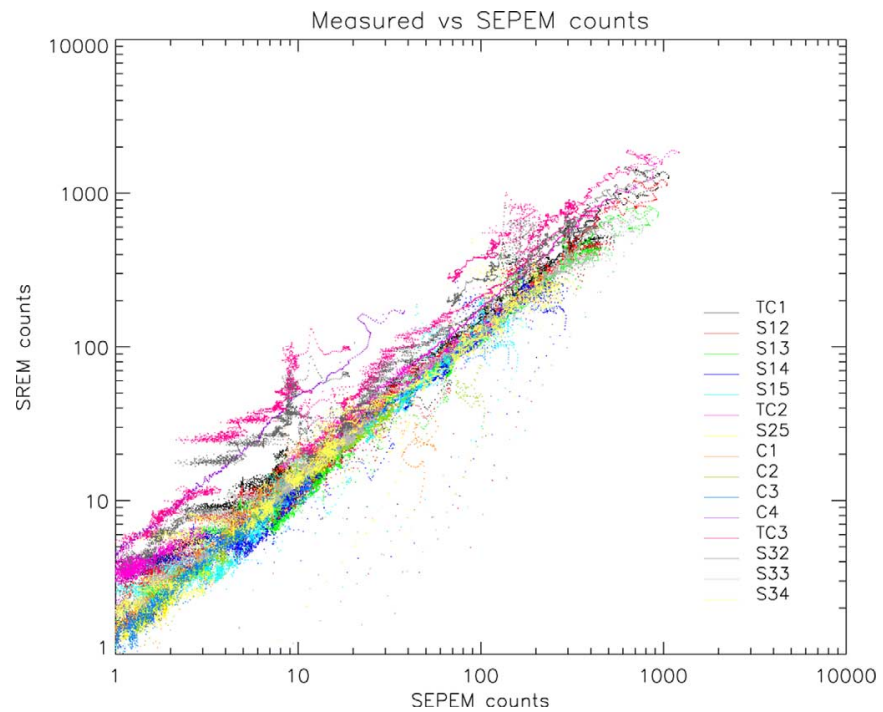

Fig. 8. Scatter plot of INTEGRAL/SREM versus SEPEM/SREM count-rates for the January 2005 SEPEs.

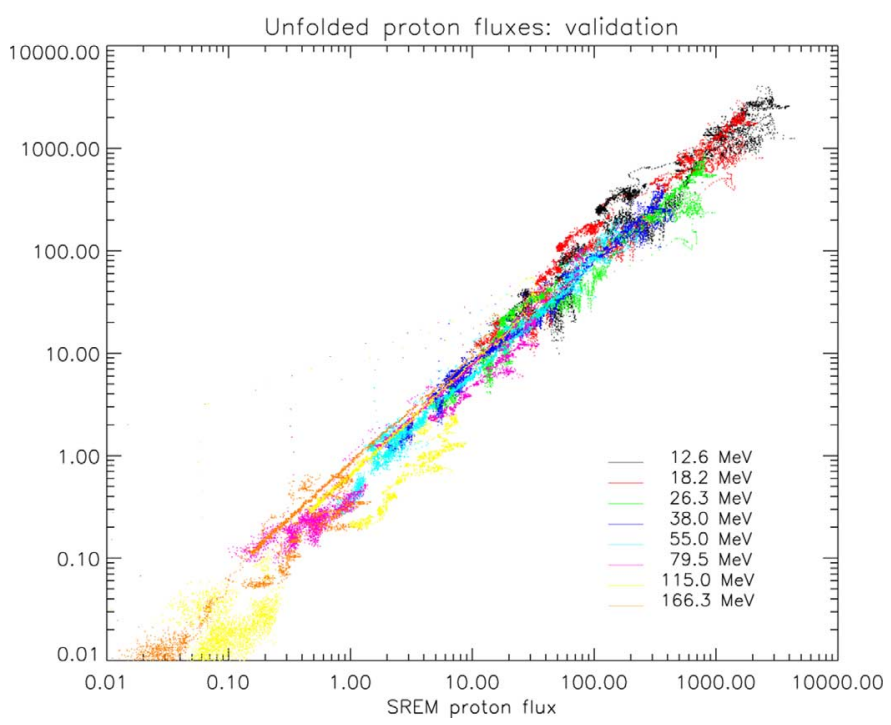

Fig. 9. Scatter plot of INTEGRAL/SREM and SEPEM proton fluxes for the January 2005 SEPEs.

It should be noted that the band covered by the count-rate scatter plot in Fig. 8 (which involves folded distributions) is wider compared to that of the associated proton fluxes scatter plot in Fig. 9 (which involves unfolded distributions). One would expect the opposite since the errors and uncertainties attributed to the unfolding method are much larger compared to the folded distributions - which are guaranteed by construction to have small residuals. This indicates that the differences in the count-rate scatter plot (Fig. 8) can be partially attributed to uncertainties in the measurements and/or to the response function of individual SREM channels. Moreover, in contrast to the SEPEM counts, a fraction of the SREM counts in Fig. 8 can be due to the incident electron fluxes. Remarkably, the unfolding procedure for the proton fluxes smears out these effects and despite the uncertainties of the inversion method the band covered by the scatter plot of proton fluxes in Fig. 9 is relatively smaller than that of Fig. 8 . The consistency between 


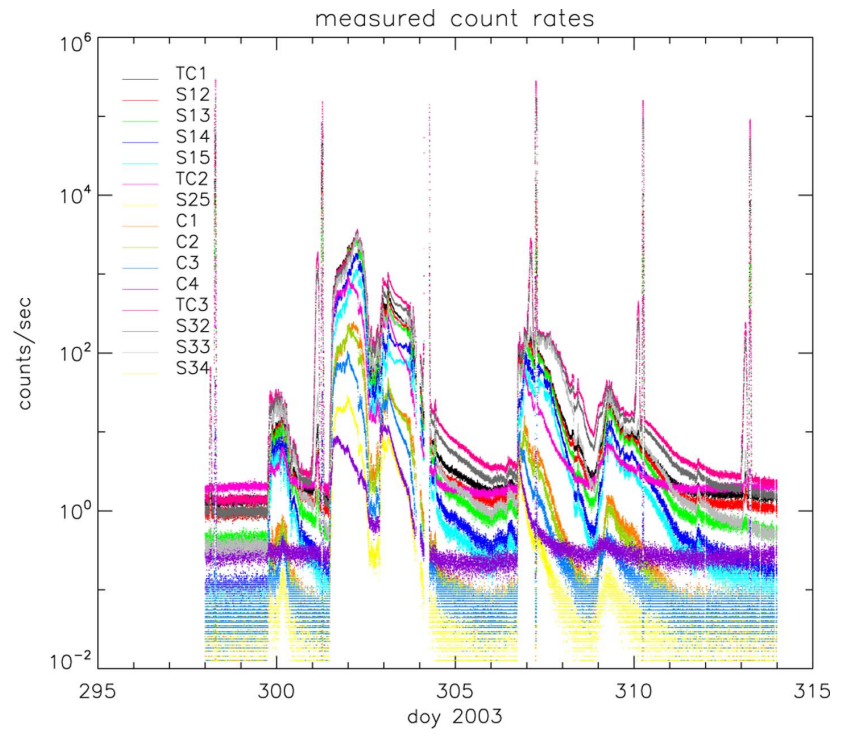

Fig. 10. Count-rates of INTEGRAL/SREM for the sequence of October-November 2003 SEPEs, versus the Day of Year (DOY) 2003.

the proton flux datasets verifies both the results of the method and the method per se at least for the unfolding of proton fluxes in proton dominated radiation environments.

The sum of the reconstructed proton count-rates exceeded the sum of the measured ones in 2165 out of the 7277 unfolded spectra. In all cases the difference was slight and a factor of about $0.8-1$ was applied to match the sum of the measured count-rates. In the rest of the cases, the sum of the reconstructed proton count-rates was less than the sum of the measured ones. The difference is presumably attributed to count-rates triggered by incident electron fluxes. Taking into account that the derived proton fluxes are consistent with the SEPEM fluxes, one can conclude that the contribution of the electron count-rates in these cases is not dominant.

In what follows, we briefly present some results using similar analysis for the measurements of the INTEGRAL/SREM unit during the occurrence of the multiple October-November 2003 SEPEs. In Fig. 10 we present the measurements by INTEGRAL/ SREM, in Fig. 11 the unfolded proton flux series derived by the developed method and interpolated at SEPEM characteristic energies, and finally a comparison plot with the SEPEM dataset is presented in Fig. 12. This scatter plot contains $11429 \times 8$ out of the $22281 \times 8$ points for the eight proton flux levels. Note, that a small correction factor of about $0.8-1$ was applied to 3352 out of the 11429 unfolded spectra in order to match the total number of the reconstructed count-rates with the measured ones. The derived proton fluxes are consistent with the SEPEM dataset for this sequence of SEPEs as well.

In order to provide additional quantitative comparison between the derived fluxes and SEPEM fluxes, we have calculated the sum $\sum \overline{f_{i}}\left(E_{i}\right)=\sum f\left(E_{i}\right) \Delta E_{i}$ for each proton energy bin. The summation is performed over the considered flux series for the selected SEPEs presented in Figs. 9 and 12. It is expected that the measured fluxes will present differences attributed to the different orbits of the spacecraft and the spatial complexity of the evolving in time solar energetic particle fluxes. In Table II

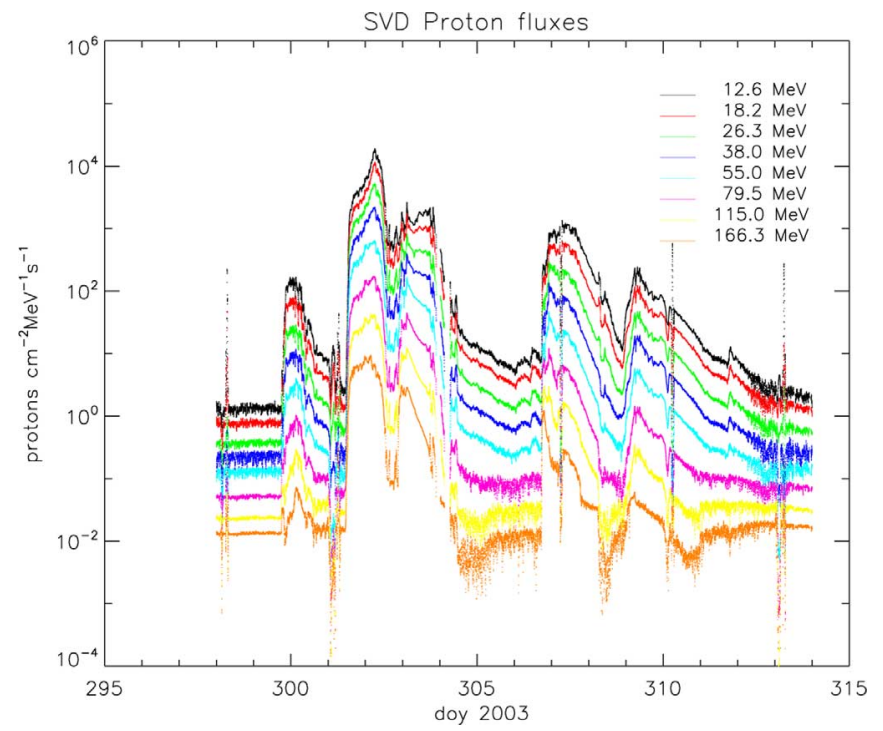

Fig. 11. The SVD-derived INTEGRAL/SREM proton flux series for the sequence of October-November 2003 SEPEs interpolated at SEPEM energy bins.

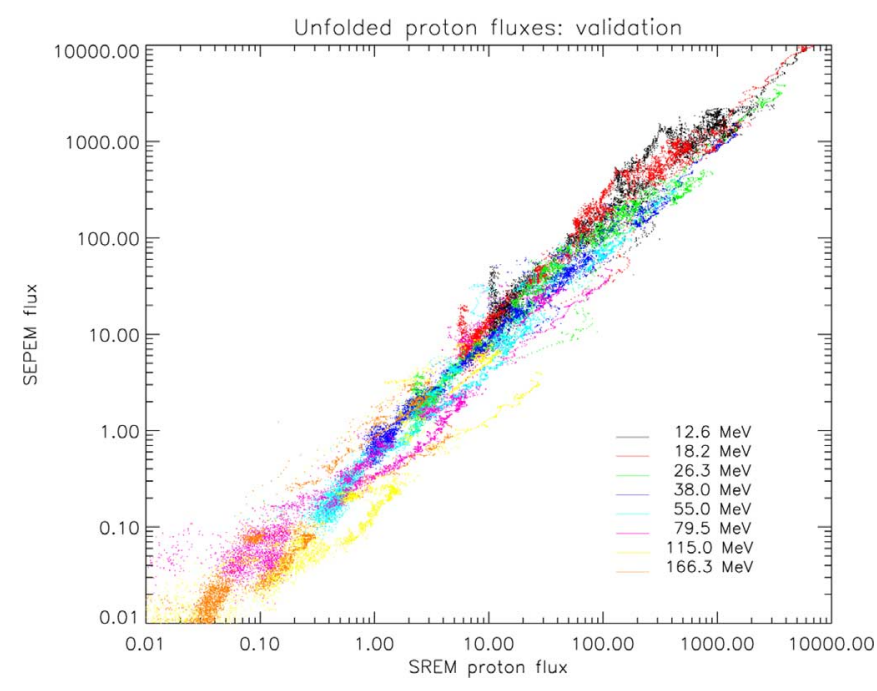

Fig. 12. Scatter plot between the unfolded and the SEPEM proton fluxes for the sequence of October-November 2003 SEPEs.

we present the values of the total fluxes of each event at different energy windows. The values between the datasets are in very good agreement with each other, considering the uncertainties of the measurements and the errors associated with the processing of both datasets.

In summary, we have compared about 20000 unfolded INTEGRAL/SREM proton flux spectra of SEPEs with associated spectra extracted from the SEPEM dataset. The comparative scatter plots indicate a very good agreement between the datasets. This finding shows that proper analysis of SREM data lead to the derivation of high energy proton fluxes of similar reliability with other processed datasets based on scientific instrument measurements.

\section{The Solar Energetic Particle Flux ToOl}

Using the method described above, we have developed a web-based tool which provides solar energetic proton flux se- 
TABLE II

TOTAL FLuXES $\sum \bar{f}\left(E_{i}\right)\left[\mathrm{p}^{+} / \mathrm{cm}^{2} / \mathrm{sec}\right]$

\begin{tabular}{|l||cc||cc|}
\hline SEPEs & DOY 2005 & $\mathbf{1 7 - 2 3}$ & DOY 2003 & 298-314 \\
\hline Ebin $_{i}[\mathrm{MeV}]$ & $\sum \bar{f}_{\text {srem }}$ & $\sum \bar{f}_{\text {sepem }}$ & $\sum \bar{f}_{\text {srem }}$ & $\sum \bar{f}_{\text {sepem }}$ \\
\hline$[10.5-15.1]$ & $1.82 \mathrm{e}+7$ & $1.90 \mathrm{e}+7$ & $6.41 \mathrm{e}+7$ & $8.95 \mathrm{e}+7$ \\
{$[15.1-21.9]$} & $1.62 \mathrm{e}+7$ & $1.75 \mathrm{e}+7$ & $5.63 \mathrm{e}+7$ & $8.27 \mathrm{e}+7$ \\
{$[21.9-31.6]$} & $1.09 \mathrm{e}+7$ & $0.75 \mathrm{e}+7$ & $3.97 \mathrm{e}+7$ & $3.53 \mathrm{e}+7$ \\
{$[31.6-45.7]$} & $7.02 \mathrm{e}+6$ & $5.53 \mathrm{e}+6$ & $2.64 \mathrm{e}+7$ & $2.33 \mathrm{e}+7$ \\
{$[45.7-66.1]$} & $3.77 \mathrm{e}+6$ & $3.00 \mathrm{e}+6$ & $1.32 \mathrm{e}+7$ & $1.13 \mathrm{e}+7$ \\
{$[66.1-95.6]$} & $1.63 \mathrm{e}+6$ & $1.16 \mathrm{e}+6$ & $5.11 \mathrm{e}+6$ & $3.27 \mathrm{e}+6$ \\
{$[95.6-138]$} & $6.74 \mathrm{e}+5$ & $4.28 \mathrm{e}+5$ & $1.92 \mathrm{e}+6$ & $7.63 \mathrm{e}+5$ \\
{$[138-200]$} & $2.76 \mathrm{e}+5$ & $2.34 \mathrm{e}+5$ & $6.20 \mathrm{e}+5$ & $3.35 \mathrm{e}+5$ \\
\hline$[10.5-200]$ & $5.86 \mathrm{e}+7$ & $5.44 \mathrm{e}+7$ & $2.07 \mathrm{e}+8$ & $2.46 \mathrm{e}+8$ \\
\hline
\end{tabular}

ries based on SREM data to the space scientific and engineering community. The Solar Energetic Proton Flux (SEPF) [12] tool displays results from various locations in space through the analysis of multi-point count-rate measurements of SREM units on-board INTEGRAL, Herschel, Planck, and Rosetta. The SEPF tool downloads SREM data [1], calculates the differential proton fluxes using the scheme presented in Section III and publishes the latest results for the current year and the most recent week on a daily basis. The fact that the developed method is linear, enables the SEPF tool to provide results in fast times. The unfolding of a single set of data requires time less than $0.2 \mathrm{sec}$ in a PC with a moderate single CPU. The SEPF tool has been developed and is operated by the Institute for Space Applications \& Remote Sensing, National Observatory of Athens (http://proteus.space.noa.gr/sepf_tool/).

\section{CONCLUSION}

A novel method for the calculation of reliable proton fluxes using measurements from SREM units was presented. The derived results:

- exhibit enhanced spectral resolution;

- successfully reconstruct the original SREM count-rates;

- are in very good agreement with processed datasets based on scientific instruments.

The proposed method provides to the scientific community a simple approach for the solution of a rather ill-defined class of inverse problems. It should be stressed, that the validity of the method has been confirmed so far for SREM proton fluxes measured in radiation environments where electron count-rates do not dominate.

The described method permits also the characterization of the SREM measurements, i.e., if they correspond to a proton or to an electron dominated environment. Using measurements during SEPEs and spacecraft crossings through proton and electron radiation belts, we have verified (not shown here) that the ratio between the sums of reconstructed proton and electron count-rates (for the D1 and D3 set of counters) provides a reliable index for the characterization of SREM measurements. However, it should be noted that further studies and a suitable electron reference dataset would be required for the validation of the developed unfolding method in electron dominated environments, since the electron response matrix of SREM units has different characteristics than that of protons.

The application of the proposed method permits the extraction of reliable proton flux series from the SREM units on-board ESA spacecraft on different orbits (LEO, MEO, IP and L2 point), which encounter different radiation environments. The construction of a solar energetic proton flux database using the measurements of multiple SREM units is expected to provide new insights in the studies of the properties of SEPEs.

\section{ACKNOWLEDGMENT}

The authors would like to thank the SEPEM project http:// sepem.aeronomie.be/ for providing access to the SEPEM application server.

\section{REFERENCES}

[1] SREM. [Online]. Available: http://srem.web.psi.ch/

[2] A. Mohammadzadeh, E. Evans, P. Nieminen, E. Daly, P. Vuilleumier, P. Bühler, C. Eggel, W. Hajdas, N. Schlumpf, A. Zehnder, J. Schneider, and R. Fear, "The ESA Standard Radiation Environment Monitor Program first results from PROBA-1 and Integral," IEEE Trans. Nucl. Sci., vol. 50, no. 6, pp. 2272-2275, 2003.

[3] H. D. R. Evans, P. Bühler, W. Hajdas, E. J. Daly, P. Nieminen, and A. Mohammadzadeh, "Results from the ESA SREM monitors and comparison with existing radiation belt models," Adv. Space Res., vol. 42, pp. 1527-1537, 2008.

[4] A. Hajdas, P. Bühler, C. Eggel, P. Favre, A. Mchedlishvili, and A. Zehnder, "Radiation environment along the INTEGRAL orbit measured with the IREM monitor," Astro. Astrophys., vol. 411, pp. L43-L47, 2003.

[5] Solar Energetic Particle Environment Modelling Dataset. [Online]. Available: http://dev.sepem.oma.be/

[6] W. Hajdas, A. Zehnder, L. Adams, and B. Nickson, "The proton irradiation facility at the Paul Scherrer Institut.," Nucl. Instrum. Methods Phys. Res. B, vol. 113, pp. 54-54, 1996.

[7] S. Agostinelli, J. Allison, and K. Amako, "GEANT4-A simulation toolkit," Nucl. Instrum. Methods Phys. Res. A, vol. 506, no. 3, pp. 250-303, 2003.

[8] Communication with Dr. Laurent Desorgher. desorgher@spaceit.ch, Space-IT GmbH, CH-3012 Bern, Switzerland.

[9] G. Cowan, Statistical Data Analysis. Oxford, U.K.: Oxford Univ. Press, 1998.

[10] A. Höcker and V. Kartvelishvili, "SVD approach to data unfolding," Nucl. Instrum. Methods Phys. Res., vol. A 372, pp. 469-481, 1996.

[11] P. C. Hansen and D. P. O'Leary, "The use of L-curve in the regularisation of discrete ill-posed problems," SIAM J. Sci. Comput., vol. 14, pp. 1487-1503, 1996

[12] Solar Energetic Particle Flux Tool. [Online]. Available: http://proteus. space.noa.gr/sepf tool/ 